\title{
Assessment of Adherence to Guidelines for Testing and Treatment of Pharyngitis Among Children in Palestine: A Retrospective Study
}

\section{Shahenaz Najjar}

Arab American University

Hiba H Falana

Birzeit University

Haya 0 Sultan

Birzeit University

Mutaz Dreidi

Birzeit University

Razan 0 Ata

Arab American University

Maryam A Manasrah

Arab American University

Hani A. Naseef ( $\nabla$ hshtaya@birzeit.edu )

Birzeit University https://orcid.org/0000-0001-9700-8532

\section{Research}

Keywords: Pharyngitis, Antibiotic, Bacterial resistance, Swab Culture, Overuse

Posted Date: November 8th, 2021

DOI: https://doi.org/10.21203/rs.3.rs-1040296/v1

License: (9) This work is licensed under a Creative Commons Attribution 4.0 International License.

Read Full License 


\section{Abstract \\ Background}

Acute Pharyngitis is a common upper respiratory tract infection, and is considered to be one of the leading causes for paediatric outpatient visits. Bacterial pharyngitis is less common and is mainly caused by Group A Beta haemolytic streptococcus (GABHS), also known as Streptococcus pyogenes. Accurate diagnosis guided by appropriate diagnostic tools is essential in managing paediatric pharyngitis as antibiotics are indicated only for documented bacterial cases. This researched aimed to assess the extent of physicians' adherence to clinical guidelines regarding the testing and management of pharyngitis among children in Palestine.

\section{Method}

A retrospective, observational study that was conducted at the Palestine Medical Complex (PMC). Data were collected from the emergency department between June and December 2019. The Modified Centor score was used to assess the extent of physicians' adherence to guidelines regarding pharyngitis testing and management among children 3 to 13 years old. It included assessing throat swab collection and antibiotics prescribing. SPSS was used for data analysis.

\section{Results}

A total of 290 cases were collected. 217 patients (74.8\%) had a Modified Centor score of $\geq 2 ; 126$ of them received antibiotics and swab was taken for only 8 of them. On the other hand, 73 cases $(25.2 \%)$ had a Modified Centor score of $<2 ; 34$ of them received antibiotics. Azithromycin was the most commonly prescribed antibiotic (41.3\%), followed by Amoxicillin-clavulanic acid (38.1\%). The frequency of empirical antibiotics prescribing was significantly higher among children with Centor score $>2$, elder children and those presenting with fever.

\section{Conclusion}

Pharyngitis management should include appropriate swab collection and antibiotic selection. Prescribing antibiotics empirically early on for most patients with pharyngitis will hasten the development of bacterial resistance and will increase the cost of treatment without adding any benefit in terms of protecting patients from pharyngitis complications.

\section{Background}

Acute Pharyngitis is a common upper respiratory tract infection, and is considered to be one of the leading causes for paediatric outpatient visits[1]. Viruses contribute to the majority of cases[2]. 
Adenovirus, respiratory syncytial virus, influenza, parainfluenza and enteroviruses are some of the common viral causes of pharyngitis [3]. Bacterial pharyngitis is less common and is mainly caused by Group A Beta haemolytic streptococcus (GABHS), also known as Streptococcus pyogenes [3]. Throat culture remains the gold standard for diagnosing GABHS pharyngitis. Rapid antigen-detection test (RADT) is another available diagnostic tool [3]. Distinguishing between viral and bacterial pharyngitis is essential in guiding therapy. Viral pharyngitis, for instance, require supportive care only while early recognition of streptococcal pharyngitis and initiating the appropriate antibiotics is crucial in decreasing the risk of possible serious complications such as acute rheumatic fever (ARF)[4]. Yet, antibiotics should not be started empirically even if GABHS pharyngitis is suspected, because it could lead to antibiotics overuse especially that initiating antibiotics within 9 days of the onset of illness still can prevent ARF [3]. Once GABHS pharyngitis is confirmed, a 10-day course of penicillin should be the first line therapy. In penicillin allergic patients, alternatives include cephalosporins (preferably first generation), clindamycin and macrolides[3]

Since pharyngitis is a common cause of paediatric outpatient visits, poor adherence to guidelines could lead to a significant unjustified antibiotic overuse. This concern is supported by the findings of several previous studies. In Lithuania for instance, pharyngitis was found to be one of the three-most common indications for prescribing antibiotics in paediatric population [5]. Moreover, in a study conducted in the United States between 1997 and 2010, the antibiotic prescription rate was found to be 60\% [6]. This is a high rate when considering that GABHS infection in school-age children is estimated to account only for $15-30 \%$ of cases [3]. In Boston, a retrospective cohort study found that over one quarter of patients aged 3-18 years were prescribed antibiotics for pharyngitis but did not require antibiotics according to guidelines [7]. Another observational retrospective study in Italy showed similar results as antibiotics were prescribed in $63.1 \%$ of the cases, while $56.5 \%$ of them were non-GABHS pharyngitis or undefined pharyngitis[8]. According to a study that was conducted in Jordan, $61 \%$ of upper respiratory tract infections were treated with antibiotics, while antibiotics were unnecessary in $86 \%$ of these cases[9].

Therefore, it is important to assess the local level of antibiotics prescribing for pharyngitis in an attempt to detect and improve malpractices. This is particularly important now as we live in an era where antibiotic resistance has become a major concern and is thought to become the leading cause of death in a couple of decades [10]. In this study we aim to assess and review the level of adherence to standard pharyngitis testing and antibiotics prescription in children aged 3 to 13 years in a central governmental hospital in Palestine.

\section{Methods}

This is a retrospective, observational study that was conducted at the Bahrain Paediatrics Hospital in the Palestine Medical Complex (PMC), a central governmental hospital in Ramallah, Palestine. This 45-bed hospital provides several services for children aged 1 day to 13 years. Pharyngitis cases that were encountered between June and December 2019 were collected. Exclusion criteria included patients who had suspension of lower respiratory tract infections or other sources of infections (e.g., urinary tract 
infections), patients who were on antibiotics at the time of presentation, having other comorbidities (e.g., congenital heart disease), patients to be admitted and those who were discharged against medical advice. Patients less than 3 years old were also excluded because the presentation of GABHS is variable and appropriate management is less defined among this age category[11]. Only cases that were clearly documented in the detailed physicians' notes as pharyngitis were included. More than 35,000 cases were registered at the emergency department during the study period, of which 537 cases had pharyngitis. After applying the exclusion criteria, a total of 290 cases were included and reviewed.

Two teams of a total of 4 researchers were responsible for data collection. All extracted data were doublechecked. The extracted data from each file were the following: demographics (patient's file number, gender, age, date of visit and presence of penicillin allergy), presenting symptoms (fever, cough, tonsillar swelling or exudate and swollen, tender anterior cervical nodes), and management (throat swab taken or not, antibiotic prescribed or not, and the type of antibiotic). Patient file numbers were kept private and used only to access the mentioned data. The Modified Centor score (or Mclsaac score) was calculated for each patient using the indicators mentioned in Table 1[12]. Microsoft Excel was used to arrange data and the Statistical Package for the Social Sciences (SPSS) was used for data analysis.

Calculation of the Modified Centor score (Mclsaac score):

The Mclsaac score was used in this study as a clinical score to assess the likelihood of having GAS pharyngitis [13]. The clinical importance of this score stems from the fact that it has sensitivity up to $96.9 \%$ compared to physician care which has sensitivity of $70.6 \%$. Moreover, specificity is similar to that compared to physician care. This score assigns points to certain clinical features to identify patients at higher risk for bacterial pharyngitis as shown in Table 1. In our study, if a patient's record indicated that they had a fever of more than 38 degrees, we deemed them to have fever. In most of the cases, fever was unspecified and the grade wasn't mentioned, therefore the patient wasn't assigned a point. Regarding the other parameters, patients scored the points according to the data documented in their files

\section{Table 1: Modified Centor (or Mclssac) Score}

\begin{tabular}{|ll|}
\hline Table 1: Modified Centor (or Mclssac) Score & \\
\hline Criteria & Points \\
\hline Temperature $>38^{\circ} \mathrm{C}\left(>100.5^{\circ} \mathrm{F}\right)$ & 1 \\
\hline Absence of cough & 1 \\
\hline Swollen, tender anterior cervical nodes & 1 \\
\hline Tonsillar swelling or exudate & 1 \\
\hline Age 3 to 14 years & 1 \\
\hline
\end{tabular}




\section{Results}

A total of 290 cases were included this study. The majority of them were males (61.4\%) and in the age range of $3-6$ years $(52 \%)$, Table 2 . Around one third of the study sample $(38 \%, n=180)$ were reported to have fever when they came to hospital. The majority of the cases did not complain of swollen cervical nodes $(97 \%, n=282)$, nor tonsils swelling $(97.6 \%, n=283)$. For cough, half of the study sample was reported to have cough $(n=145)$. More than two thirds of the cases $(75 \%, n=217)$ scored two or more on the Modified Centor Score Criteria $(n=217)$ Table 3, of which $58 \%$ received antibiotics $(n=126)$. For the 73 cases that had a Modified Centor score of $<2,34$ of them ( $46.6 \%$ received antibiotics and no swab was taken for any of them. Overall, it was found that more than half the study sample $(55.2 \%, n=160)$ were prescribed antibiotics. Azithromycin was found to be the most commonly prescribed antibiotic $(23.4 \%, n=68)$ followed by Amoxicillin/ clavulanic acid $(20 \%, n=58)$ Table 4 . For throat swab, eight patients had their throat swab taken (2.8\%), all of which had a Centor score of more than two, and 6 of them empirically received antibiotics. Out of the 160 cases that received antibiotics, only two patients had a documented penicillin allergy and were given azithromycin accordingly. Over the seven-month period, pharyngitis visits were most common in December, followed by September, with least number of cases in June (Figure 1). Among the included variables, it was found that there is a significant correlation between Modified Centor Score value and antibiotic prescribing $(r=0.154, p=0.009)$. This indicates that the chance of receiving antibiotic was higher for those presenting with more signs and symptoms. Presence of fever was also found to be significantly associated with antibiotic prescription $(r=0.204, p<0.001)$. Furthermore, age was found to be an important variable as more antibiotics were prescribed for elder children compared to younger children $(r=0.189, p=0.001)$. Lastly, the rate of antibiotic prescribing was not found to be altered by the time of the year at which a case was seen ( $p$ value: 0.520 )

\section{Table 2. Demographics of cases $(\mathrm{N}=290)$.}

\begin{tabular}{|lll|}
\hline Variable & $\mathbf{N}$ & $\%$ \\
Gender & & \\
Male & 178 & $61.4 \%$ \\
Female & 112 & $38.6 \%$ \\
\hline Age & & \\
$3-6$ years & 151 & $52 \%$ \\
$7-10$ years & 87 & $30 \%$ \\
$11-13$ years & 52 & $18 \%$ \\
\hline
\end{tabular}

Table 3: Modified Centor Score (MSC) Results 


\begin{tabular}{|lll|}
\hline Variable & $\mathbf{N}$ & $\%$ \\
\hline MCSC* & & \\
1 & 73 & $25.2 \%$ \\
2 & 166 & $57.2 \%$ \\
3 & 50 & $17.2 \%$ \\
4 & 1 & $0.3 \%$ \\
\hline * MCSC: Modified Centor Score Criteria. \\
\hline
\end{tabular}

Table 4. Prescribed Antibiotics $(\mathrm{N}=290)$.

\begin{tabular}{|lll|}
\hline Antibiotics & N & $\%$ \\
\hline No antibiotics & 130 & $44.8 \%$ \\
\hline Azithromycin & 68 & $23.4 \%$ \\
\hline Amoxicillin & 25 & $8.6 \%$ \\
\hline Amoxicillin/Clavulanic Acid & 58 & $20 \%$ \\
\hline Cefixime & 2 & $0.7 \%$ \\
\hline Ceftriaxone + Amoxicillin/Clavulanic Acid & 2 & $0.7 \%$ \\
\hline Cefdinir & 1 & $0.3 \%$ \\
\hline Cefuroxime & 1 & $0.3 \%$ \\
\hline Penicillin & 2 & $0.7 \%$ \\
\hline Cefadroxil & 1 & 0.3 \\
\hline
\end{tabular}

\section{Discussion}

This is the first objective study to assess antibiotics prescribing and physicians' adherence to clinical guidelines regarding the management of pharyngitis in paediatric population in Palestine. The results revealed that throat swabs are not used routinely in the studied setting which led to expected overprescribing of antibiotics. Moreover, inappropriate antibiotic selection was observed as well. As indicated by the results, around three-quarters of the cases had a Modified Centor score of $\geq 2$. According 
to clinical guidelines, a Modified Centor score of $\geq 2$ necessitates testing for GABHS pharyngitis using throat swab or RADT, as the likelihood for GABHS pharyngitis in patients having a Modified Centor score of 2 is estimated to be $20.5 \%$ and increases as the score increases[14]. Antibiotic therapy should be started once GABHS pharyngitis is documented by RADT or throat culture. However, only 8 throat cultures were obtained, yet 160 patients (55\%) received antibiotics empirically, without any confirmatory test for the presence of bacterial pharyngitis. Throat swabs are an essential part of pharyngitis management, and their use is encouraged in various clinical guidelines to properly guide therapy and decrease unnecessary antibiotics use [15].

Our findings revealed that $74.8 \%$ of patients had a score of $\geq 2$, and more than half of them received antibiotics without having any confirmatory tests for GABHS pharyngitis (throat culture was ordered only for 8 of them). This is an alarming finding and would lead to significant overuse of antibiotics. As previous studies showed that only $25 \%$ of patients with a Centor score of $\geq 3$ had a documented positive throat culture for GABHS pharyngitis [16]. On the other hand, even patients having a score $<2$, half of them received antibiotics, while no throat swabs were ordered for these patients. However, it was found that the higher the Centor score is the higher the chance of antibiotic prescribing $(r=0.154, p=0.009)$. This indicates that the more signs and symptoms the child experience, the more likely physicians will attribute it to bacterial causes. This emphasizes the vital role of throat cultures, as a certain diagnosis can't be made relying only on clinical features. Moreover, in the absence of a positive throat culture or RADT, overestimation of bacterial pharyngitis and the associated antibiotic use remain a true concern.

In this study fever was found to be the most common complaint occurring in one third of the study sample and was significantly associated with antibiotics prescribing. This finding is in agreement with other studies where fever was reported to be the most common clinical feature in confirmed bacterial cases [16]. However, fever is also seen in viral pharyngitis and decisions made solely based on the presence of fever might lead to antibiotics overuse.

In pharyngitis, similar to other infections, there might be certain alarming information demanding and justifying the use of empirical antibiotics before obtaining swab results. These include limited conditions where a patient has a high suspicion of GABHS pharyngitis; i.e. having a diagnosis of scarlet fever, a symptomatic child having a household member with documented GABHS infection or in cases where the patient (or a family member) has a history of ARF [3].

However, if none of the mentioned criteria is present, diagnosis should always be confirmed using throat culture or RADT prior to antibiotic initiation [3]. This is a safe practice even when MCS is $>2$ (i.e GABHS is suspected) since starting antibiotics in bacterial pharyngitis within 9 days of symptom onset can still prevent the development of complications (mainly rheumatic fever) $[3,17]$.

Regarding antibiotic selection, there was an obvious inappropriate antibiotic selection, similar to previous findings involving paediatric patients [18]. In a study that was conducted in Canada, for example, antibiotics were prescribed inappropriately in $85 \%$ of cases in preschool children having pharyngitis [8]. Another study from the United states showed that over a quarter of children received antibiotics for 
pharyngitis, although they weren't indicated according to treatment guidelines[7]. This indicates that antibiotic overprescribing is a global issue facing the healthcare sector, not only in our studied setting. Oral penicillin $\mathrm{V}$ or amoxicillin are recommended in treating GABHS pharyngitis and should be selected as first line agents for patients without penicillin allergy. These agents have a narrow spectrum of activity, few side effects and are of reasonable cost [19]. Macrolides and cephalosporins can be used as alternatives for type I and II penicillin allergies, respectively [3]. However, as shown by Table 4, azithromycin was prescribed for 66 patients (41\%) whereas only 2 patients had a documented penicillin allergy. These findings are similar to other studies' findings where macrolides use in pharyngitis was reported to be substantially increasing against the recommendations of treatment guidelines [18]. Physicians are advised to adhere to clinical guidelines regarding antibiotic selection as there is no evidence that macrolides are clinically superior to penicillin in the absence of penicillin allergy [20].

Pharyngitis cases can be seen anytime throughout the year, but peak seasons depend on the causative agent, and it is generally during winter[3,21,22]. This matches our results (Fig. 1), as the highest incidence was in December. However, pharyngitis cases due to viral causes, are usually seen in summer $[2,21]$. This could explain the gradual increase of visits from June to September as shown in Chart 1. However, when it comes to antibiotics prescribing, the rate of antibiotic prescribing was not found to be altered by the time of the year at which a case was seen ( $p$ value: 0.520 ). This could indicate that little attention is being payed to viral pharyngitis as a possible and common cause for pharyngitis especially in summer season. Another interesting finding is that antibiotic prescribing rate was significantly higher in elder children than younger children which might be out of fear of developing serious complications such as ARF. Overall, the results of this study highlight the need for enforcing committing to guidelines in the management of pharyngitis.

\section{Conclusion}

The results of this study strongly emphasize the need for reviewing and adhering to clinical guidelines. It was noticed that throat swabs are not used routinely in the studied setting which led to expected overprescribing of antibiotics. Moreover, inappropriate antibiotic selection was observed as well, necessitating modifying the usual prescribing patterns using evidence-based medicine. Rationalizing the use of antibiotics has become one of the main health related goals in the past years. While it is still challenging to do so in some medical departments or for some critical cases for which treatment is not yet standardized, it is essential to adhere to clinical guidelines for those infectious diseases for which treatment has been well established. Pharyngitis management should include appropriate swabs collection and antibiotics selection. Prescribing antibiotics empirically early on for most patients with pharyngitis will hasten the development of bacterial resistance and will increase the cost of treatment without additional benefit in terms of protecting patients from severe pharyngitis complications.

\section{Strengths And Limitations Of This Study}


Strengths: The study was conducted in a central governmental hospital where most of the nearby population receives medical help. The hospital hosts paediatric residency program, so the study reflects the practice of current and future paediatricians.

Limitations: Only 7 months were included since ICD-10 codes were not properly used and cases collection through electronic search was not feasible. Data were collected manually through reviewing all ER cases and it is therefore subjected to human errors.

\section{Abbreviations}

Beta haemolytic streptococcus (GABHS), Rapid antigen-detection test (RADT), Acute Rheumatic Fever (ARF), Palestine Medical Complex (PMC)

\section{Declarations}

\section{Ethics approval}

The study was approved by the IRB committee at Faculty of Graduate Studies, Health Informatics Program, Health Sciences Department, Arab American University.

\section{Consent for publication}

Not applicable.

\section{Data Availability:}

Data available from the corresponding author upon reasonable request.

\section{Competing interests}

The authors state that they do not present any conflict of interests in the present research

\section{Funding:}

This research received no specific grant from any funding agency in the public, commercial or not-forprofit sectors.

\section{Authors' contributions}

All authors have made valuable contributions to this study. SN designed the study, planned and supervised the work, review -editing, HF, HS designed the study, did the literature search, drafted the manuscript, participated in data analysis, editing, MD, analyzed the results, editing, RA, MM, collected the data, HN, review, editing, and submission of the article. All co-authors approved the final version of the manuscript. 


\section{Acknowledgments}

We would like to express our heartfelt gratitude to all health team at Palestine Medical Complex for their support.

\section{References}

1. Gerber MA. Pharyngitis. Princ Pract Pediatr Infect Dis [Internet]. Elsevier; 2008 [cited 2021 Feb 25]. p. 206-13. Available from: https://linkinghub.elsevier.com/retrieve/pii/B9780702034688500353

2. Bower JR. Pharyngitis. Netter's Infect Dis. Elsevier Inc.; 2012. P. 177-82.

3. Tanz Rr. Nelson Textbook Of Pediatrics. 20th Ed. Richard E. Behrman M, editor. Elsevier; 2016.

4. Pichichero M. Treatment and prevention of streptococcal pharyngitis in adults and children UpToDate. 2020. Accessed 28 Sept 2021.

5. Karinauske E, Kasciuskeviciute S, Morkuniene V, Garuoliene K, Kadusevicius E. Antibiotic prescribing trends in a pediatric population in Lithuania in 2003-2012: Observational study. Medicine (Baltimore). NLM (Medline); 2019;98:e17220.

6. Dooling KL, Shapiro DJ, Van Beneden C, Hersh AL, Hicks LA. Overprescribing and inappropriate antibiotic selection for children with pharyngitis in the United States, 1997-2010. JAMA Pediatr. American Medical Association; 2014. p. 1073-4.

7. Brennan-Krohn T, Ozonoff A, Sandora TJ. Adherence to guidelines for testing and treatment of children with pharyngitis: A retrospective study. BMC Pediatr. BioMed Central Ltd.; 2018;18:43.

8. Barbieri E, Donà D, Cantarutti A, Lundin R, Scamarcia A, Corrao G, et al. Antibiotic prescriptions in acute otitis media and pharyngitis in Italian pediatric outpatients. Ital J Pediatr. BioMed Central Ltd.; 2019;45.

9. Al-Omari M, Yahia G, Khader Y, Batieha A, Dauod AS. Antibiotics in Upper Respiratory Tract Infections: Appropriateness of the Practice in Jordan. Jordan Med J [Internet]. 2011 [cited 2021 Oct 25];44:4139. Available from: https://journals.ju.edu.jo/JMJ/article/view/2081

10. WHO. New report calls for urgent action to avert antimicrobial resistance crisis. 2019.

11. Shulman ST, Bisno AL, Clegg HW, Gerber MA, Kaplan EL, Lee G, et al. Clinical Practice Guideline for the Diagnosis and Management of Group A Streptococcal Pharyngitis: 2012 Update by the Infectious Diseases Society of America. Clin Infect Dis [Internet]. Oxford Academic; 2012 [cited 2021 Oct 20];55:e86-102. Available from: https://academic.oup.com/cid/article/55/10/e86/321183

12. Fine AM, Nizet $\mathrm{V}$, Mandl KD. Large-scale validation of the centor and mcisaac scores to predict group A streptococcal pharyngitis. Arch Intern Med. 2012;172:847-52.

13. Mclsaac WJ, Kellner JD, Aufricht P, Vanjaka A, Low DE. Empirical Validation of Guidelines for the Management of Pharyngitis in Children and Adults. J Am Med Assoc. 2004;291:1587-95.

14. Line F. Pearls For The Management Of Pharyngitis Www.Rxfiles.Ca @ Sept 2019 Empiric Drug Regimens Of Choice \& Susceptibility Concerns Pharyngitis: Management Considerations. 2019;7- 
11.

15. Gunnarsson R, Ebell MH, Wächtler H, Manchal N, Reid L, Malmberg S, et al. Association between guidelines and medical practitioners' perception of best management for patients attending with an apparently uncomplicated acute sore throat: a cross-sectional survey in five countries. BMJ Open. British Medical Journal Publishing Group; 2020;10:e037884.

16. Balasubramanian, Amperayani S, Dhanalakshmi K, Senthilnathan S, Chandramohan V. Rapid antigen diagnostic testing for the diagnosis of group A beta-haemolytic streptococci pharyngitis. Natl Med J India. Medknow Publications and Media Pvt. Ltd.; 2018;31:8.

17. Treatment and prevention of streptococcal pharyngitis in adults and children - UpToDate.

18. Dooling KL, Shapiro DJ, Beneden C Van, Hersh AL, Hicks LA. Overprescribing and Inappropriate Antibiotic Selection for Children With Pharyngitis in the United States, 1997-2010. JAMA Pediatr. American Medical Association; 2014;168:1073-4.

19. AAFP. Practice Guidelines IDSA Updates Guideline for Managing Group A Sreptococcal Pharyngitis. Am Fam Physician. 2013;

20. Satti AM, Alkenany HA, Alsafi MY, Hussain MA. Efficacy of Macrolides versus Penicillins against Streptococcal pharyngitis : a meta-analysis of randomized controlled ... Efficacy of Macrolides versus Penicillins against Streptococcal pharyngitis: a meta-analysis of randomized controlled trials. 2016;

21. Gereige R, Cunill-De Sautu B. Throat infections. Pediatr Rev. 2011;32:459-69.

22. Brkic FF, Besser G, Janik S, Gadenstaetter AJ, Parzefall T, Riss D, et al. Peaks in online inquiries into pharyngitis-related symptoms correspond with annual incidence rates. Eur Arch Oto-RhinoLaryngology. Springer Berlin Heidelberg; 2020;1-8.

\section{Figures}

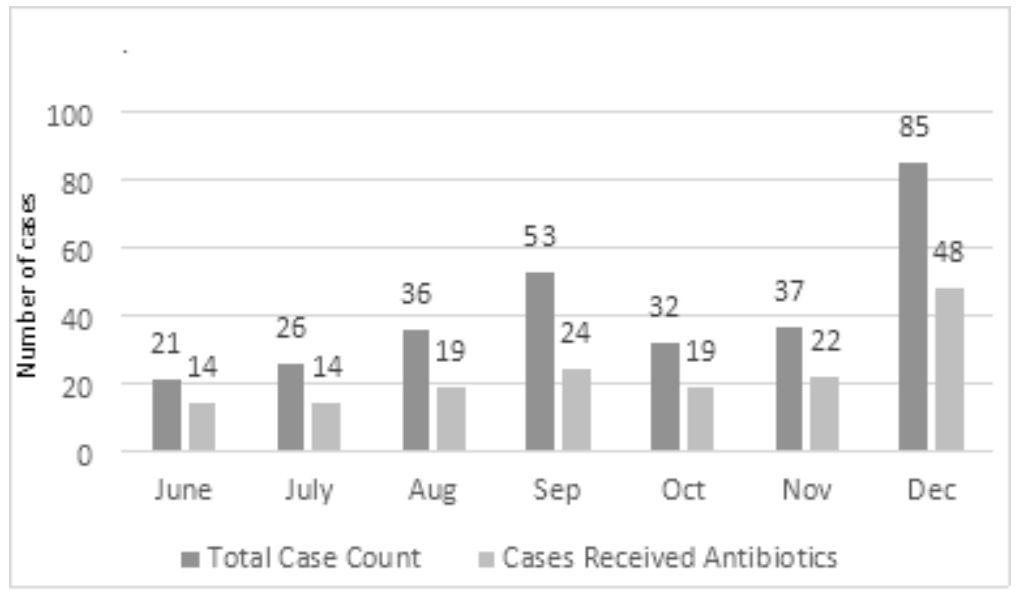

\section{Figure 1}

Cases distribution over the selected months 